\title{
Nonlinear Analysis of Chatter Phenomena Generated in a Bicycle Disc Brake*
}

\author{
Takashi NAKAE**, Takahiro RYU*** and Atsuo SUEOKA**** \\ **Department of Mechanical Engineering, Toyama National College of Technology \\ 13 Hongo, Toyama-shi, Toyama 939-8630, Japan \\ E-mail: nakae@nc-toyama.ac.jp \\ ${ }^{* * *}$ Department of Mechanical and Energy Systems Engineering, Oita University \\ 700 Dannoharu, Oita-shi, Oita 870-1192, Japan \\ ****International Research Center for Hydrogen Energy, Kyushu University \\ 744 Motooka, Nishi-ku, Fukuoka-shi, Fukuoka 819-0395, Japan
}

\begin{abstract}
Squeal and chatter phenomena are often generated in bicycle disc brakes. The squeal is in-plane unstable vibration along the disc surface caused by dry friction with negative slope with respect to the relative velocity. The squeal is generated in the vibrating system including the brake unit, spokes and hub. According to experiments, the chatter is generated in a certain limited range at high disc temperatures, and is nonlinear frictional vibration in which the out-of-plane unstable vibration of the disc due to Coulomb friction and the squeal are combined through the internal resonance relationship between the out-of-plane and in-plane vibrations caused by the increase in disc temperature during braking. First, we propose a simple nonlinear system with three degrees of freedom which possesses the essential vibration factors included in a bicycle disc brake system to investigate whether or not the coupled nonlinear vibration due to the internal resonance occurs. Then, we numerically confirm the mechanism of the chatter generated in a bicycle disc brake by nonlinear analysis.
\end{abstract}

Key words: Self-excited Vibration, Frictional Vibration, Nonlinear Vibration, Unstable Vibration, Dry Friction, Coulomb Fiction, Chatter, Squeal, Bicycle Disc Brake

\section{Introduction}

It has been found experimentally in previous studies ${ }^{(1),(2)}$ that two types of frictional self-excited vibrations are generated in bicycle disc brakes during braking: squeal with frequency of around $1 \mathrm{kHz}$ and chatter with frequency of around $500 \mathrm{~Hz}$. Squeal is mainly in-plane vibration along the disc surface caused by dry friction with negative slope with respect to the relative velocity, referred to simply as dry friction in this report. Chatter is a nonlinear phenomenon in which out-of-plane unstable vibration due to Coulomb friction perpendicular to the disc surface and in-plane unstable vibration (i.e., squeal) are combined through internal resonance in a certain limited range at high disc temperatures.

We analyzed the squeal of bicycle disc brakes that was generated in the experiment by applying the characteristic value problem to a proposed linear analytical vibration model ${ }^{(3)}$. This analytical model of the bicycle disc brake system was devised to reproduce the experimental results for a coupled out-of-plane and in-plane vibrating system composed of the disc, hub, caliper and spokes. The decrease in the out-of-plane natural frequencies of the disc with increasing disc temperature was taken into account in the analysis. As a result, it was found that two types of unstable vibrations were generated in the bicycle disc brake

${ }^{*}$ Received 19 Apr., 2010 (No. 10-0168) [DOI: 10.1299/jsdd.4.632]

Copyright (C) 2010 by JSME 
system. One was caused by dry friction that generates torsional vibration of the disc and hub in the in-plane direction with the frequency of around $1 \mathrm{kHz}$. In a limited range at high disc temperatures, the other vibration was generated mainly in the out-of-plane vibration mode of the disc caused by Coulomb friction with frequency of around $500 \mathrm{~Hz}$, according to the linear analysis ${ }^{(3)}$. However, since the frequency ratio between the unstable vibrations was approximately $2: 1$ in a simple integer ratio, coupled nonlinear vibration due to the internal resonance relationship between them, that is, the chatter investigated in this report, can occur in a limited high temperature range if the nonlinear vibration characteristics included in the analytical model are taken into account. In previous researches ${ }^{(4),(5)}$, there have been many cases where the internal resonance between the vibration modes generated in the same direction was considered. However, the chatter investigated here is a new case where the internal resonance between vibration modes is generated in different directions.

First, by using a simple coupled vibration model of a block subjected to friction, we confirm that the coupled nonlinear vibration due to internal resonance between vibration modes, such as chatter generated in the bicycle disc brake system, occurs. Then, the nonlinear internal resonance phenomenon known as chatter, is analyzed by newly considering the nonlinear frictional characteristics of the bicycle disc brake system treated in the previous report ${ }^{(3)}$.

\section{Characteristics of Chatter ${ }^{(1),(2)}$}

Squeal is generated in bicycle disc brake systems. Although the disc temperature increases up to $300{ }^{\circ} \mathrm{C}$ during long braking, the squeal frequency remains constant at around $1 \mathrm{kHz}$. On the other hand, the chatter is generated in the high temperature range of 260 to $290{ }^{\circ} \mathrm{C}$ only when squeal occurs as the disc temperature increases. The frequency of chatter in the out-of-plane direction of the disc has a constant value of around $500 \mathrm{~Hz}$. The frequency ratio of squeal to chatter is fixed exactly at 2:1. The spectrum of chatter includes the squeal frequency as a second-order harmonic component.

Figure 1 shows the out-of-plane chatter mode of the disc, as measured by a scanning laser Doppler vibrometer. The chatter mode is fixed in space while the disc rotates. The small squares represent the measuring points. The white and black squares represent the points at which the values of vibration velocity are largely out-of-phase. The pad is located at a node of the chatter mode on the disc, and the number of the nodal radii denoted by gray arrows is 5 (odd). In chattering, the amplitude of the out-of-plane vibration of the disc is remarkably large.

Figure 2 shows a schematic diagram of the coupled out-of-plane natural mode $\mathrm{D}^{(2),(3)}$. The mode is the same as the chatter mode of the disc shown in Fig. 1. During braking, the frequency of mode $\mathrm{D}$ passes through $500 \mathrm{~Hz}$ (half of the squeal frequency of around $1 \mathrm{kHz}$ ) in the disc temperature range of 260 to $290{ }^{\circ} \mathrm{C}$. Consequently, the internal resonance phenomenon between the out-of-plane unstable vibration of the disc due to Coulomb friction and the in-plane unstable vibration (i.e., squeal) of the disc and hub is generated in this disc temperature range. Hence, the frequency ratio of squeal to the out-of-plane

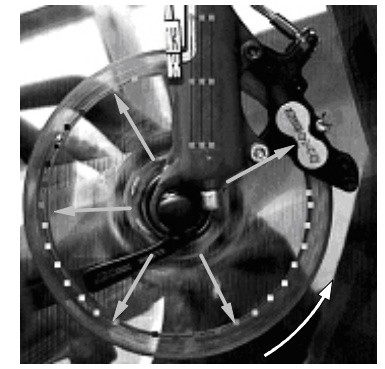

Fig. 1 Out-of-plane chatter mode of disc ${ }^{(1)}$

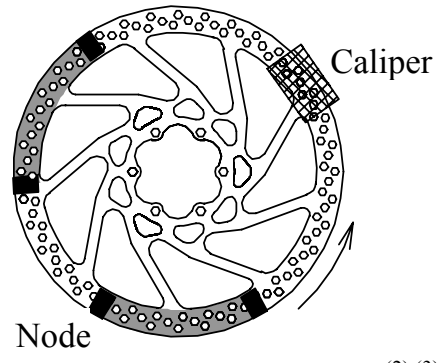

Fig. 2 Coupled natural mode $\mathrm{D}^{(2),(3)}$ 


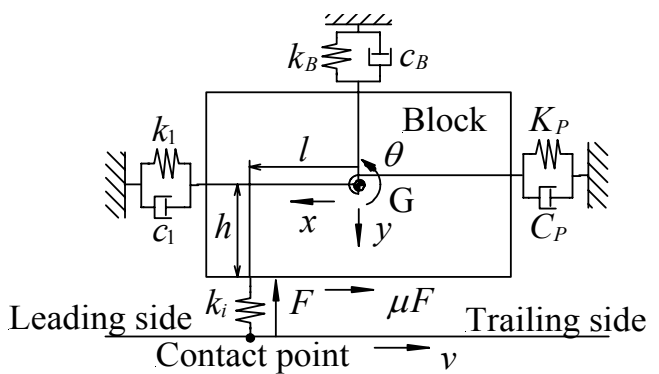

Fig. 3 Analytical model subjected to friction

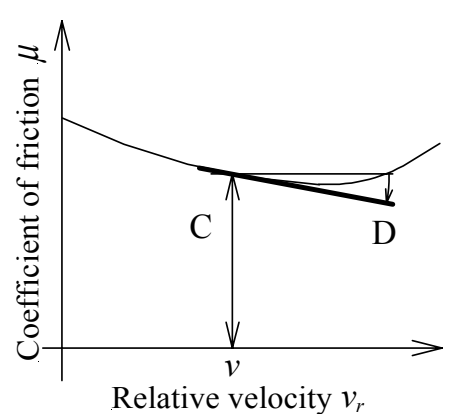

Fig. 4 Characteristics of friction

unstable vibration of the disc is fixed exactly at 2:1, and considerable nonlinear vibration occurs as a result. This is the presumed generation mechanism of chatter.

\section{Theoretical Analysis of Vibrating System with Three Degrees of Freedom}

\subsection{Analytical model and equations of motion}

Before we describe the nonlinear analytical results for the chatter generated in a bicycle disc brake system, a simple analytical model is proposed to investigate whether or not the coupled nonlinear vibration due to the internal resonance occurs in this section. This model possesses the essential vibration factors included in the bicycle disc brake system.

Figure 3 shows the analytical model with three degrees of freedom subjected to friction. The linear and angular displacements of the block are represented by $x, y$ and $\theta$. The rigid block can vibrate in the plane motion in the $x$-direction and in the out-of-plane motion in the $y$ - and $\theta$-directions. The center of gravity of block $\mathrm{G}$ is supported by spring $k_{1}$ and dashpot $c_{1}$ in the $x$-direction, by spring $k_{B}$ and dashpot $c_{B}$ in the $y$-direction, and by rotational spring $K_{P}$ and rotational dashpot $C_{P}$ in the $\theta$-direction. The block is placed on a horizontal surface moving at the constant velocity of $v$; the block slips in contact with the surface at a point shifted from the center of gravity by $l$ in the $x$-direction and by $h$ in the $y$-direction. The contact point is modeled by spring $k_{i}$ with only rigidity.

Figure 4 shows the characteristics of friction acting at the contact point. The abscissa is the relative velocity $v_{r}$ and the ordinate is the coefficient of friction $\mu$. The characteristics of friction have two essential factors, namely, the magnitude of the coefficient of friction (denoted by $\mathrm{C}$ ) and the negative slope (indicated by D) at a certain relative velocity $v$. The former causes the out-of-plane vibration in the direction perpendicular to the frictional force and hence has the effect of so-called Coulomb friction. The latter causes the in-plane vibration in the direction of the frictional force due to dry friction. Here, we consider the coupled out-of-plane and in-plane vibrations under such characteristics of friction, taking the internal resonance between the vibrations into account.

The contact force $F$ acting on the block is expressed as

$$
F=k_{i}(y+l \theta)+F_{0},
$$

where $F_{0}$ is the static pressing load. We take the coefficient of friction $\mu$ with negative slope with respect to relative velocity $v_{r}$ in the $x$-direction between block and floor surface as the following nonlinear function:

$$
\mu=e_{0}-e_{1} v_{r}+e_{2}\left(v_{r}-v\right)^{3},
$$

where $e_{0}, e_{1}$ and $e_{2}$ are positive constants. Equation (2) describes so-called constant Coulomb friction when $e_{1}=e_{2}=0$, whereas dry friction is found when $e_{1}>0$. On the other hand, the slope of the coefficient of friction with respect to relative velocity becomes positive and increases in the high relative velocity range, as shown in Fig. 4. We confirmed that the characteristics of friction obtained from the experiment by using bench test apparatus $^{(2)}$ showed also the feature of the expression in Eq.(2).

The relative velocity $v_{r}$ in the $x$-direction is expressed as 


$$
v_{r}=v+\dot{x}-h \dot{\theta} .
$$

As seen in Eq. (3), the relative velocity is expressed as a function of the variables $\dot{x}$ and $\dot{\theta}$ with respect to the in-plane and the out-of-plane vibrations, respectively; therefore, the in-plane and out-of-plane vibrations are coupled.

The equations of motion of the rigid block are represented by

$$
\begin{aligned}
& m \ddot{x}+c_{1} \dot{x}+k_{1} x=-\mu F, \\
& m \ddot{y}+c_{B} \dot{y}+k_{B} y=-F, \\
& J \ddot{\theta}+C_{P} \dot{\theta}+K_{P} \theta=-F l+\mu F h,
\end{aligned}
$$

where $m$ is the mass of the block, and $J$ is the moment of inertia of the block about its axis of rotation through its center of gravity. The nonlinear vibration characteristics are included in the term $\mu F$ on the right hand side of Eqs. (4) and (6).

For the linear analysis $\left(e_{2}=0\right)$, by arranging the coupled equations with respect to the fluctuating quantities in the in-plane and out-of-plane directions, and linearizing the resulting equations, the following linear differential equations are obtained:

$$
\begin{aligned}
\boldsymbol{M} \ddot{\boldsymbol{x}}+\boldsymbol{C} \dot{\boldsymbol{x}}+\boldsymbol{K} \boldsymbol{x}=\mathbf{0}, & {\left[\begin{array}{l}
x \\
y \\
\theta
\end{array}\right], \boldsymbol{M}=\left[\begin{array}{ccc}
m & 0 & 0 \\
0 & m & 0 \\
0 & 0 & J
\end{array}\right], \boldsymbol{C}=\left[\begin{array}{ccc}
-F_{0} e_{1}+c_{1} & 0 & F_{0} h e_{1} \\
0 & c_{B} & 0 \\
F_{0} h e_{1} & 0 & -F_{0} h^{2} e_{1}+C_{P}
\end{array}\right], } \\
\boldsymbol{K} & =\left[\begin{array}{ccc}
k_{1} & \varepsilon k_{i} & \varepsilon k_{i} l \\
0 & k_{B}+k_{i} & k_{i} l \\
0 & k_{i}(l-\varepsilon h) & K_{P}+k_{i} l(l-\varepsilon h)
\end{array}\right],
\end{aligned}
$$

where $\varepsilon=e_{0}-e_{1} v(>0)$ corresponds to the magnitude of the coefficient of friction $\mathrm{C}$ shown in Fig. 4.

Here, the vector $\boldsymbol{x}$ can be rearranged such that the fluctuating variables have no unidirectional values. One of the diagonal elements in the damping matrix $\boldsymbol{C}$ can be negative, for example, the term surrounded by a red line, because of the dry friction that generates unstable vibration in the direction of frictional force, which is one of the mathematical mechanisms of the squeal generation. In addition, the stiffness matrix $\boldsymbol{K}$ is asymmetric $^{(6)}$ because of the presence of Coulomb friction $(\varepsilon>0)$. The out-of-plane unstable vibration can be generated because the two terms surrounded by blue lines become different in sign as $\varepsilon$ becomes large. This is the other mathematical mechanism of unstable vibration caused by Coulomb friction that generates mainly the out-of-plane vibration perpendicular to the frictional force. In this way, the effects of dry friction and Coulomb friction are both present in the analytical model. From the features of the stiffness matrix, the in-plane vibration is subject to the influence of the out-of-plane vibration unidirectionally.

Transforming Eq. (7) into a coupled linear first-order ordinary differential equation, the stability can be numerically analyzed as a characteristic value problem. If a real part of the characteristic values is positive at least, then the system is unstable; that is, unstable vibration occurs. The imaginary part of the characteristic value with the positive real part corresponds to the angular frequency of the unstable vibration.

For the nonlinear analysis $\left(e_{2}>0\right)$, the shooting $\operatorname{method}^{(7)}$ is used to solve the periodic self-excited vibration caused by friction. To set the initial phase of the resulting self-excited vibration, $\dot{\theta}=0$ is adopted for applying the shooting method.

\subsection{Parameters for calculation}

The parameter values used in the numerical computation are listed in Table 1. To represent the magnitude of spring constants $k_{i}, k_{B}$ and $K_{P}$, and the damping coefficient $C_{P}$, the following frequencies $f_{i}, f_{B}$ and $f_{P}$, and the damping ratio $\zeta_{P}$ are defined as follows: 
Table 1 Values of parameters

\begin{tabular}{c|c||c|c||c|c}
\hline \hline$F_{0}$ & $157 \mathrm{~N}$ & $J$ & $8.52 \times 10^{-4} \mathrm{~kg} \mathrm{~m}$ & $f_{i}$ & $170 \mathrm{~Hz}$ \\
\hline$e_{0}$ & 0.4 & $l$ & $0.010 \mathrm{~m}$ & $f_{B}$ & $300 \mathrm{~Hz}$ \\
\hline$e_{1}$ & $0.056 \mathrm{~s} / \mathrm{m}$ & $h$ & $0.032 \mathrm{~m}$ & $c_{1}$ & $4.7 \mathrm{~N} \cdot \mathrm{s} / \mathrm{m}$ \\
\hline$e_{2}$ & $0.06 \mathrm{~s}^{3} / \mathrm{m}^{3}$ & $v$ & $0.9 \mathrm{~m} / \mathrm{s}$ & $c_{B}$ & $23 \mathrm{~N} \cdot \mathrm{s} / \mathrm{m}$ \\
\hline$m$ & $0.555 \mathrm{~kg}$ & $k_{1}$ & $1.04 \times 10^{7} \mathrm{~N} / \mathrm{m}$ & $\zeta_{P}$ & 0.012 \\
\hline
\end{tabular}

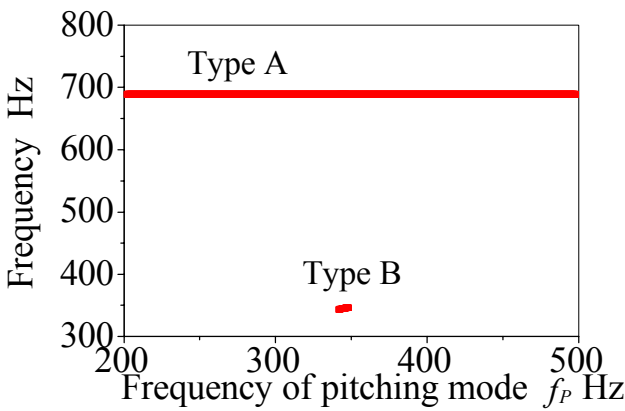

Fig. 5 Relationship between $f_{P}$ and the frequency of unstable vibration from linear analysis

Table 2 Characteristic values of Types A and B at $f_{p}=343 \mathrm{~Hz}$

\begin{tabular}{c|c|c}
\hline \hline Type & Real part s & Imaginary part $\mathrm{Hz}$ \\
\hline A & 3.626 & 689.0 \\
B & 1.511 & 343.8 \\
\hline
\end{tabular}

$$
f_{i}=\frac{1}{2 \pi} \sqrt{\frac{k_{i}}{m}}, f_{B}=\frac{1}{2 \pi} \sqrt{\frac{k_{B}}{m}}, f_{P}=\frac{1}{2 \pi} \sqrt{\frac{K_{P}}{J}}, \quad \zeta_{p}=\frac{C_{p}}{2 \sqrt{J K_{p}}},
$$

where $f_{P}$ is the frequency of the pitching mode of the block and is taken as a variable in this report.

For the parameters listed in Table 1, we can ensure numerically that $\varepsilon>0$, $-F_{0} e_{1}+c_{1}<0,-F_{0} h^{2} e_{1}+C_{p}>0, \quad l-\varepsilon h<0$ and $K_{p}+k_{i} l(l-\varepsilon h)>0$ [cf. Eq. (8)].

\subsection{Computational results}

First, the results from the linear analysis are described. The ranges in which unstable vibrations occur are indicated by the red line in Fig. 5, where the relationship between the frequency $f_{P}$ and the imaginary part of the unstable characteristic value converted to frequency is shown under the condition that spring constants $k_{1}, k_{B}$ and $k_{i}$ are constant, whereas $K_{p}$ is variable. As shown in Fig. 5, two types of unstable vibrations are found. The first has an almost constant frequency of $689.0 \mathrm{~Hz}$, independent of $f_{P}$, while the other has a frequency of around $344 \mathrm{~Hz}$ and occurs in a limited frequency range of $f_{P}=342-347$ $\mathrm{Hz}$. Hereafter, the former is referred to as Type A, and the latter is referred to as Type B.

From the analytical results, the positive real part of the characteristic value increases for Type A as the value of $e_{1}$ becomes large; therefore, Type A is caused by dry friction and mainly has a vibration mode in the $x$-direction. On the other hand, the positive real part of the characteristic value increases for Type $\mathrm{B}$ as the value of $\varepsilon=e_{0}-e_{1} v$ becomes large. Thus, Type B is caused by Coulomb friction which generates mainly the out-of-plane vibration perpendicular to the frictional force.

Table 2 shows the unstable characteristic values of Types A and B at $f_{p}=343 \mathrm{~Hz}$ in Fig. 5, where both types coexist according to the linear analysis.

Types A and B are both generated simultaneously in a certain narrow limited frequency range. However, each unstable vibration may not occur individually in this range. The strong coupled nonlinear vibration due to the internal resonance between Types A and B may occur only in this range because the frequency ratio of Type A to Type B is almost 2:1 


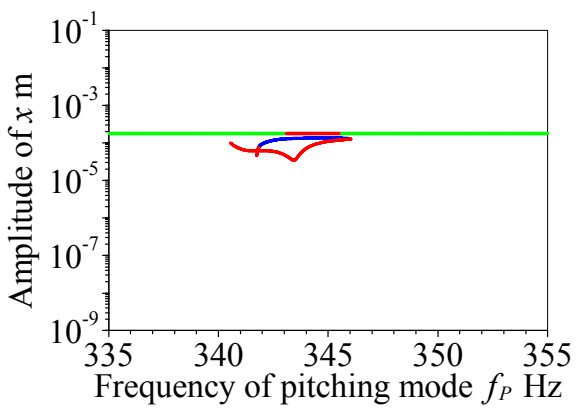

(a) Amplitude of $x$

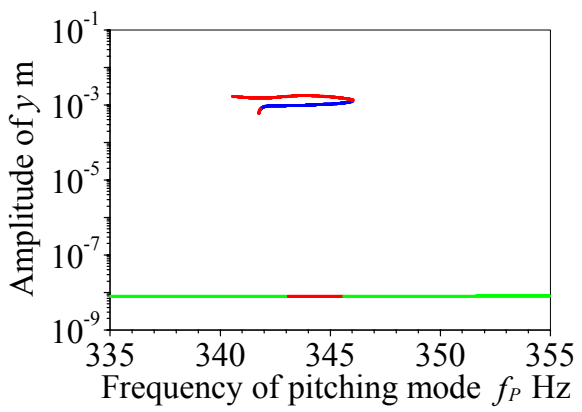

(b) Amplitude of $y$

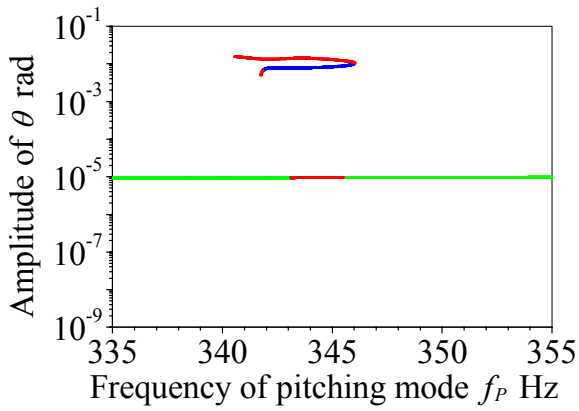

(c) Amplitude of $\theta$

Fig. 6 Relationship between $f_{P}$ and the maximum amplitudes of periodic solutions
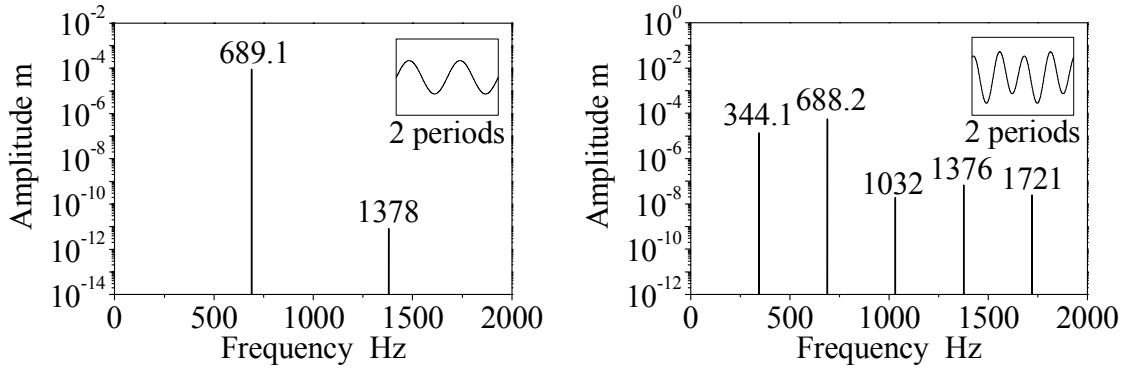

Nonlinear waveform and frequency analyses of $x$
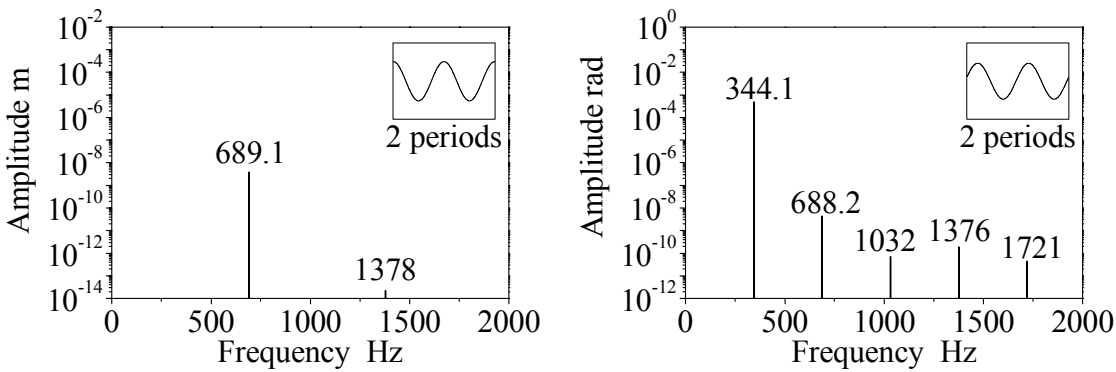

Nonlinear waveform and frequency analyses of $y$
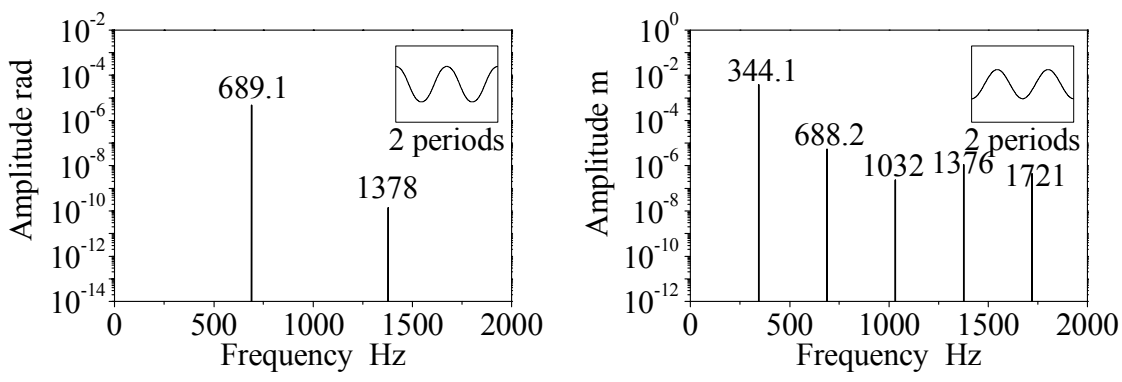

Nonlinear waveform and frequency analyses of $\theta$

(a) At $f_{P}=340 \mathrm{~Hz}$ on green line

(b) At $f_{P}=343 \mathrm{~Hz}$ on blue line

Fig. 7 Nonlinear waveforms and frequency analyses 
in a simple integer ratio. The values of parameters listed in Table 1 were adjusted in order to reproduce almost the same situation as in the bicycle disc brake system ${ }^{(3)}$.

Next, the generation of coupled nonlinear vibration due to the internal resonance is examined by the nonlinear vibration analysis, and the results of this nonlinear analysis are described. Figure 6 shows the maximum amplitudes obtained from the shooting method for $x, y$ and $\theta$, where the amplitudes are computed with changing $f_{P}$. Green and blue lines are stable periodic solutions and the red line is an unstable solution. Because we focus on only stable solutions, only the branches with stable solutions are shown for simplicity. Figure 7 shows the waveforms of $x, y$ and $\theta$, and the frequency analyses at $f_{P}=340$ and $343 \mathrm{~Hz}$ on the green and the blue lines, respectively.

In Fig. 6, there are two types of stable periodic solutions. The green stable branch is largely independent of the frequency $f_{P}$, and the amplitude in the $x$-direction is much larger than the amplitudes in the $y$ - and $\theta$-directions. The green branch is partly unstable within the frequency range of $f_{P}$ in which the blue stable branch exists. The frequency of the green branch is $689.1 \mathrm{~Hz}$ as shown in Fig. 7(a) which is almost equal to the frequency of Type A obtained from the linear analysis. Hence, the green branch corresponds to Type A in Fig. 5, and is the vibration mainly in the $x$-direction. The green branch is observed over a wide range of $f_{P}$, but has a limited unstable range in the nonlinear analysis.

The other periodic solution indicated by blue line exists in a limited frequency range of $f_{P}=342-346 \mathrm{~Hz}$, which falls within the generation range of Type B, as shown in Fig. 5, and the maximum amplitudes in the $x$-, $y$ - and $\theta$-directions on the blue branch are around $4 / 5,10^{5}$ and $10^{3}$ times those on the green branch, respectively. This means that the blue branch is coupled in both the in-plane and out-of-plane directions in the limited frequency range and the amplitudes in the $y$ - and $\theta$-directions are especially large. Type B in Fig. 5 is the out-of-plane unstable vibration caused by Coulomb friction, and hence, Type B has very small amplitude in the $x$-direction. From the difference between the vibration characteristics of the blue branch in Fig. 6 and Type B in Fig. 5, Type B cannot be realized by itself in a limited range where the frequency ratio of Type A to Type B is almost 2:1, because the coupled nonlinear vibration in the in-plane and out-of-plane directions is generated due to the internal resonance between Types A and B.

The fundamental frequency of $344.1 \mathrm{~Hz}$ in Fig. 7(b) is almost equal to that of Type B obtained from the linear analysis, as shown in Table 2. The frequency of $688.2 \mathrm{~Hz}$ in Fig. 7(b) for the in-plane vibration is included in the spectrum as the second-order harmonic component. The frequency ratio of the out-of-plane vibration to the in-plane is fixed exactly at 2:1, as shown in Fig. 7(b). Therefore, it is clear that the coupled in-plane and out-of-plane nonlinear vibrations due to internal resonance occur.

\section{Nonlinear Analysis of Bicycle Disc Brake ${ }^{(3)}$}

Figure 8 shows the analytical model of a bicycle disc brake system taken from Ref. (3). In Fig. 8(a), the rotating disc is modeled as the thin three-step stainless steel circular disc ${ }^{(8)}$, and the inner and outer pads are modeled as elastic areas with distributed contact springs and dashpots which are arranged in 19 lines over a circumferential angle of $28^{\circ}$ in 5 rows at regular intervals of $\tilde{r}_{i} / R=0.903,0.927,0.951,0.976$, and 1.00 on both the inner and outer disc surfaces, where $\left(\tilde{r}_{i}, \tilde{\theta}_{i}\right)$ are the coordinates of the $i$-th contact point on the third circular disc and $R$ is the radius of the disc. The pads are in contact with the third circular disc from the inner side. As shown in Fig. 8(b), the caliper block has two parts, namely, the blocks on inner and outer sides, and the outer block is supported on the front fork. Both blocks are connected with four bolts modeled by the spring $k_{c}$ and dashpot $c_{c}$, and with the moment spring $K^{\prime \prime}$ and moment dashpot $C^{\prime \prime}$. Figure 8(c) shows the arrangement of the disc, hub and spokes. Each spoke is assumed to be under tensile force in the longitudinal direction. A total 32 spokes $S$ and $S^{\prime}$ are separately and equally (16 each of $S$ and $S^{\prime}$ ) 

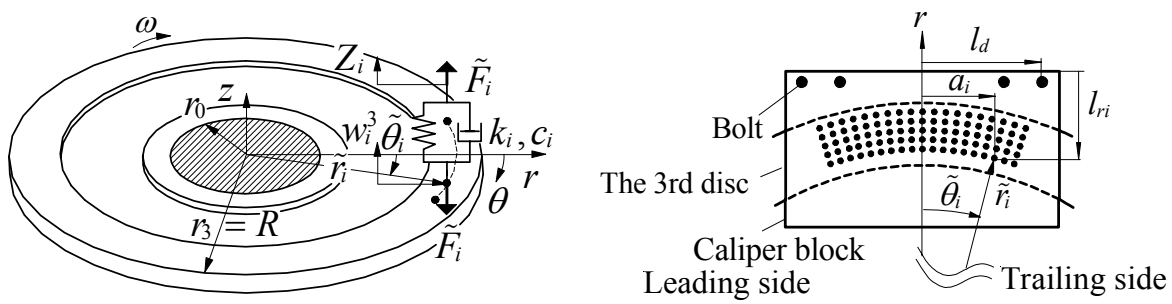

(a) Three-step circular disc and modeling of pad consisting of many contact points

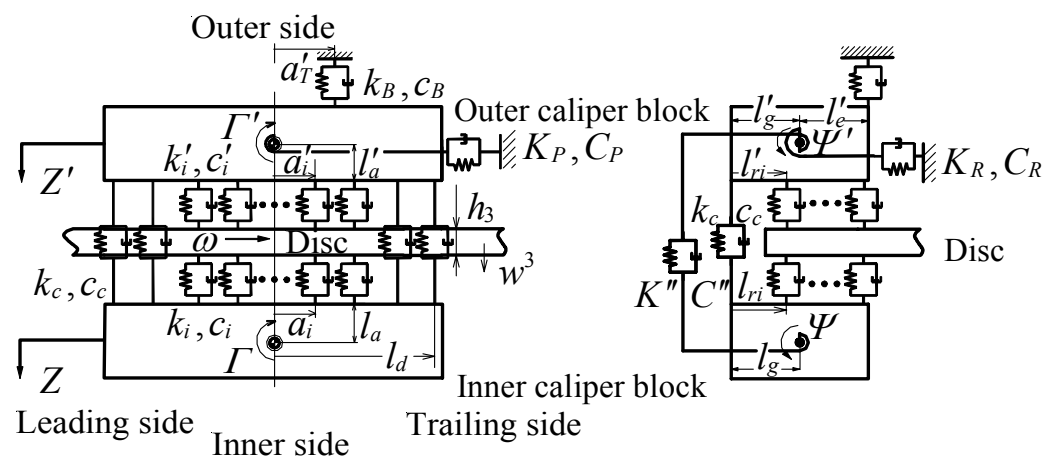

(b) Model of caliper, and top and side views of caliper

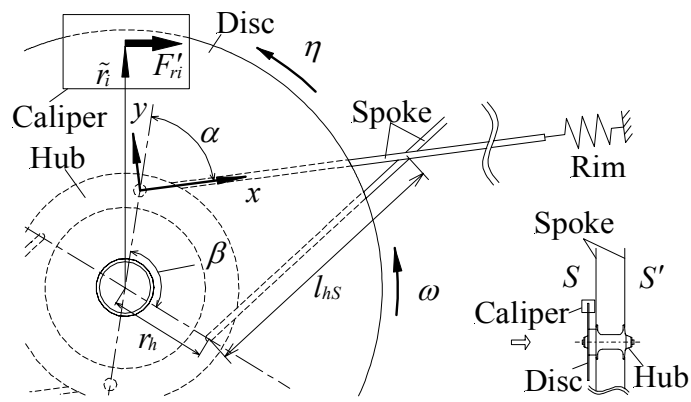

(c) Geometrical relationship among disc, hub and spokes

Fig. 8 Analytical model of disc, pad and caliper, and physical quantities and dimensions ${ }^{(3)}$

installed between the hub on the disc side and rim, and between the hub on the opposite side of disc and rim, respectively.

First, the differences between the linear analysis reported in Ref. (3) and the presentnonlinear analysis are briefly explained. Essentially, the nonlinear terms are included only in the frictional forces that act in the bicycle disc brake system. Thus, the equation of motion of a rotating circular disc, the equations of torsional motion of a hub and a disc, the equations of flexural motion of spokes, and the equations of rigid body motion of caliper blocks are all formally the same as those given in Ref. (3); thus, a detailed description is omitted here.

We again assume a coefficient of friction $\mu_{i}\left[\mu_{i}^{\prime}\right]$ at the $i$-th contact point with negative slope with respect to the relative velocity $v_{r i}\left[v_{r i}^{\prime}\right]$ in the circumferential direction at the pad between the third circular disc and caliper by using the following third-order polynomial functions in order to consider the nonlinearity of the frictional force:

$$
\mu_{i}=e_{0}-e_{1} v_{r i}+e_{2}\left(v_{r i}-\tilde{r}_{i} \omega\right)^{3}, \mu_{i}^{\prime}=e_{0}-e_{1} v_{r i}^{\prime}+e_{2}\left(v_{r i}^{\prime}-\tilde{r}_{i} \omega\right)^{3},
$$

where $\omega$ is the angular velocity of the disc, $e_{0}, e_{1}$ and $e_{2}$ are all positive constants, and the relative velocity is expressed as

$$
\left[\begin{array}{l}
v_{r i} \\
v_{r i}^{\prime}
\end{array}\right]=\left.\tilde{r}_{i}(\omega+\dot{\eta}) \mp\left[\begin{array}{l}
l_{a} \dot{\Gamma} \\
l_{a}^{\prime} \dot{\Gamma}^{\prime}
\end{array}\right] \mp \frac{h_{3}}{2}\left(\frac{\partial}{\partial t}+\omega \frac{\partial}{\partial \theta}\right) \frac{\partial w^{3}}{r \partial \theta}\right|_{r=\tilde{r}, \theta=\tilde{\theta}_{i}} .
$$

Physical quantities and dimensions with a prime ['] denote those for the outer disc surface and the outer caliper block, whereas physical quantity and dimensions without a 
prime represent those on the inner disc surface and the inner caliper block. $h_{3}$ is the thickness of the circular disc that is third from the inner side, $\eta$ is the torsional angle of the hub and disc in the in-plane direction along the disc surface, $\Gamma$ is the pitching angle of the inner caliper in the out-of-plane direction perpendicular to the disc surface, $w^{3}=w^{3}(r, \theta, t)$ is the out-of-plane displacement of the third circular disc, $t$ is time, and $r$ and $\theta$ are the radius and circumferential coordinates of the disc, respectively. The parameter $l_{a}\left[l_{a}^{\prime}\right]$ is the distance between the center of gravity of caliper block and contact surface [cf. Fig. 8(b)]. The contact force $F_{i}\left[F_{i}^{\prime}\right]$ acting at the $i$-th contact point on the disc is expressed as

$$
\left[\begin{array}{c}
F_{i} \\
F_{i}^{\prime}
\end{array}\right]=\left[\begin{array}{c}
c_{i} \\
c_{i}^{\prime}
\end{array}\right]\left\{\left.\left(\frac{\partial}{\partial t}+\omega \frac{\partial}{\partial \theta}\right) w_{i}^{3}\right|_{r=\tilde{n}_{i}, \theta=\tilde{\theta}_{i}}-\left[\begin{array}{c}
\dot{Z}_{i} \\
\dot{Z}_{i}^{\prime}
\end{array}\right]\right\}+\left[\begin{array}{c}
k_{i}\left(w_{i}^{3}-Z_{i}\right) \\
k_{i}^{\prime}\left(w_{i}^{3}-Z_{i}^{\prime}\right)
\end{array}\right],
$$

where $c_{i}\left[c_{i}^{\prime}\right]$ is damping coefficient and $k_{i}\left[k_{i}^{\prime}\right]$ is the contact spring on the pads, $w_{i}^{3}$ and $Z_{i}\left[Z_{i}^{\prime}\right]$ are the out-of-plane displacements at the $i$-th contact point on the third circular disc and on the caliper blocks expressed as

$$
Z_{i}=Z+a_{i} \Gamma-\left(l_{r i}-l_{g}\right) \Psi, \quad Z_{i}^{\prime}=Z^{\prime}+a_{i}^{\prime} \Gamma^{\prime}-\left(l_{r i}^{\prime}-l_{g}^{\prime}\right) \Psi^{\prime},
$$

respectively. $Z\left[Z^{\prime}\right], \Gamma\left[\Gamma^{\prime}\right]$ and $\Psi\left[\Psi^{\prime}\right]$ are the displacement and the rotational angles of inner [outer] caliper block related to the bouncing, pitching and rotational modes, respectively. The dimensions $a_{i}\left[a_{i}^{\prime}\right], l_{r i}\left[l_{r i}^{\prime}\right]$ and $l_{g}\left[l_{g}^{\prime}\right]$ are shown in Fig. 8(b). Consequently, the nonlinear terms included in the frictional force $\mu_{i} F_{i}\left[\mu_{i}^{\prime} F_{i}^{\prime}\right]$ are taken into account in the nonlinear analysis of the chatter.

The flexural rigidity of the disc varies with disc temperatures, which causes the decrease of the out-of-plane natural frequencies of the disc with increasing temperature. In the computation, the effect of increasing disc temperature $(T)$ during braking was taken into consideration by decreasing the longitudinal elastic modulus of the third circular disc $\left(E_{3}\right)$ in the same manner as in Ref. (3), which caused the decrease of natural frequencies of the disc in the out-of-plane direction. The following relationship is applied to the numerical analysis:

$$
E_{3}=1.41 \times 10^{6} \times(T-353)^{2}+2.06 \times 10^{10} \quad(0 \leq T \leq 350),
$$

where the units of $E_{3}$ and $T$ are $\mathrm{Pa}$ and ${ }^{\circ} \mathrm{C}$, respectively. This means that the disc has a longitudinal elasticity modulus of stainless steel $(177 \mathrm{GPa})$ at $20^{\circ} \mathrm{C}$ and the value decreases with temperature up to $353{ }^{\circ} \mathrm{C}$. By applying the function, it is confirmed that the frequency of the coupled natural mode of the disc with five nodal radii during braking (see Fig. 2), that is, the frequency of mode D shown in Fig. 1 in Ref. (3), passes through half of the squeal

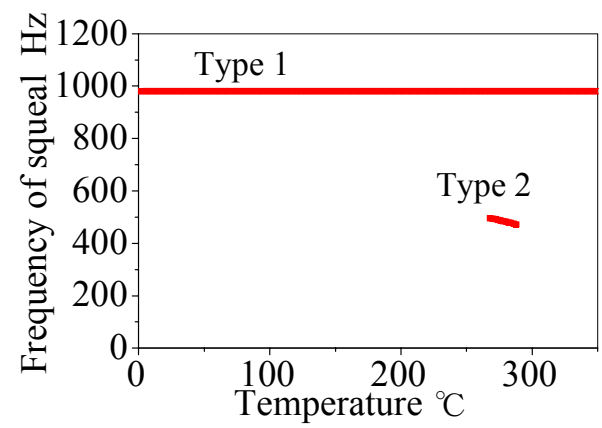

Fig. 9 Relationship between disc temperature and frequency of squeal

Table 3 Characteristic values of Types 1 and 2 at 20 and $274^{\circ} \mathrm{C}$

\begin{tabular}{c|c|c|c}
\hline \hline Temperature ${ }^{\circ} \mathrm{C}$ & Type & Real part s $^{-1}$ & Imaginary part $\mathrm{Hz}$ \\
\hline 20 & 1 & 3.730 & 980.5 \\
\hline \multirow{2}{*}{274} & 1 & 3.740 & 980.5 \\
& 2 & 0.8448 & 489.2 \\
\hline
\end{tabular}




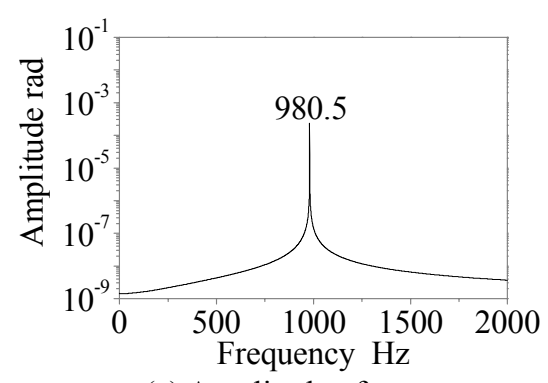

(a) Amplitude of $\eta$

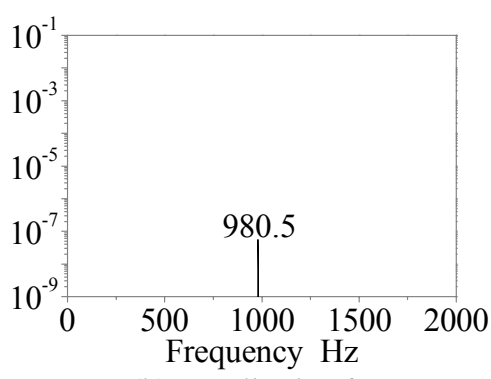

(b) Amplitude of $\Gamma$

Fig. 10 Frequency analyses at $20^{\circ} \mathrm{C}$

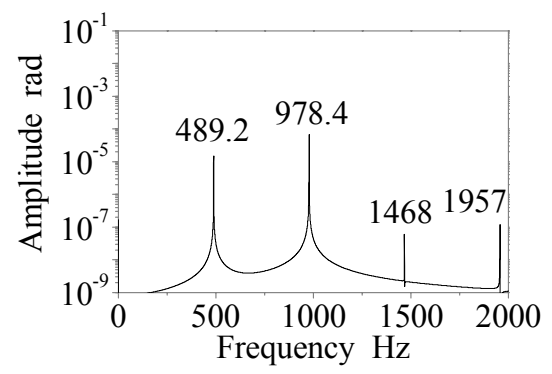

(a) Amplitude of $\eta$

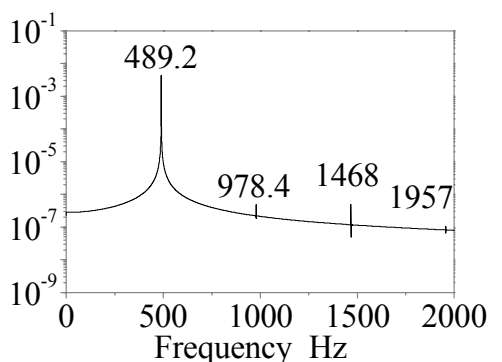

(b) Amplitude of $\Gamma$

Fig. 11 Frequency analyses at $274{ }^{\circ} \mathrm{C}$

frequency at around $280{ }^{\circ} \mathrm{C}$.

In Ref. (3), it was also evident from the linear analysis of the bicycle disc brake system that two types of unstable vibrations, referred to as Types 1 and 2, were caused by dryfriction and Coulomb friction, respectively, as in the model with three degrees of freedom analyzed in Section 3. Figure 9 shows the ranges of the unstable vibration that is generated in the bicycle disc brake system as the disc temperature increases, where the horizontal axis is the disc temperature and the vertical axis is the imaginary part of the unstable characteristic value in $\mathrm{Hz}$. Here, the constants included in the characteristics of friction are set to $e_{0}=0.32, \quad e_{1}=0.039 \mathrm{~s} / \mathrm{m}$ and $e_{2}=0.0 \mathrm{~s}^{3} / \mathrm{m}^{3}$ in order to take into account the chatter with high frequencies generated primarily in the out-of-plane direction and in only the range of high disc temperatures. Since the other numerical parameters used in the calculation are same as those used in Ref. (3), their indication is omitted here. In addition, Table 3 shows the characteristic values for Type 1 at $20^{\circ} \mathrm{C}$ and for Type 2 at 20 and $274{ }^{\circ} \mathrm{C}$ obtained from the linear analysis. The frequency ratio of Types 1 to 2 is approximately $2: 1$ at $274{ }^{\circ} \mathrm{C}$. Next, we carry out nonlinear analysis for the unstable vibrations of Types 1 and 2 generated in the linear analysis (see Fig. 9).

The Runge-Kutta-Gill method is used in the numerical calculation for the nonlinear analysis, and the periodic solutions are computed from certain initial conditions.

Numerical computation is performed for the disc temperature range of 20 to $350{ }^{\circ} \mathrm{C}$. The coefficient of dry friction $e_{2}$ is set to $0.1 \mathrm{~s}^{3} / \mathrm{m}^{3}$. Figures 10 and 11 show the frequency analyses of the torsional angle $\eta$ and the pitching angle $\Gamma$ obtained from the waveforms of the periodic solution at the disc temperatures of 20 and $274{ }^{\circ} \mathrm{C}$, respectively. The magnitudes of the in-plane and out-of-plane vibrations are typified by $\eta$ and $\Gamma$, respectively.

As shown in Fig. 10, the frequency of $\eta$ and $\Gamma$ is $980.5 \mathrm{~Hz}$ at $20^{\circ} \mathrm{C}$. This frequency is equal to that of Type 1 obtained from the linear analysis (see Fig. 9 and Table 3). The amplitude of $\eta$ is approximately $10^{3}$ times as large as that of $\Gamma$. In other words, the solution at $20^{\circ} \mathrm{C}$ in Fig. 10 corresponds to Type 1 (squeal) in Fig. 9, and has primarily the torsional vibration mode in the in-plane direction as obtained in the linear analysis. Clearly, Type 1 can be analytically generated in the nonlinear analysis as well. 


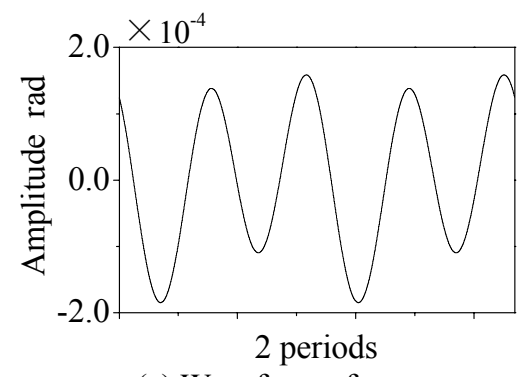

(a) Waveform of $\eta$

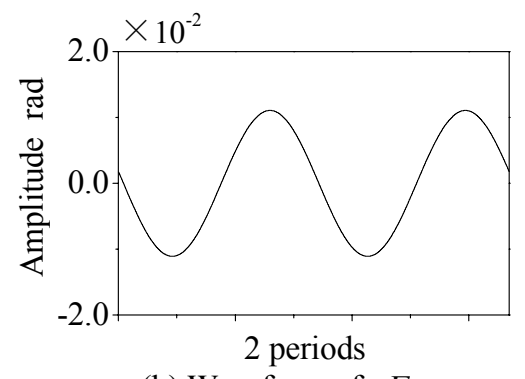

(b) Waveform of $\Gamma$

Fig. 12 Waveforms of $\eta$ and $\Gamma$ in chatter at $274{ }^{\circ} \mathrm{C}$

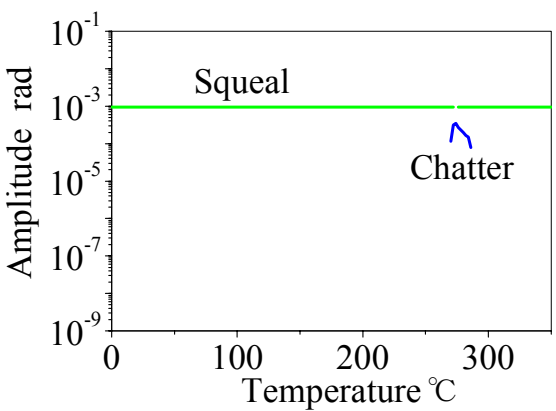

(a) Maximum amplitude of $\eta$

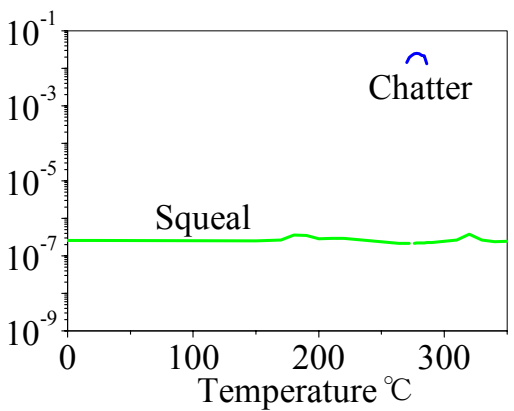

(b) Maximum amplitude of $\Gamma$

Fig. 13 Relationship between temperature and amplitudes of $\eta$ and $\Gamma$

From the results of the nonlinear analysis at $274{ }^{\circ} \mathrm{C}$ (Fig. 11), both $\eta$ and $\Gamma$ exhibit nonlinear vibration characteristics with a first-order fundamental harmonic of $489.2 \mathrm{~Hz}$ and a second-order harmonic of $978.4 \mathrm{~Hz}$ and so on. Although the corresponding fundamental frequency is equal to the frequency of Type 2, the second-order harmonic frequency differsslightly from the frequency of Type 1 (Table 3). Furthermore, the amplitude at 978.4 $\mathrm{Hz}$ is around the same level as that at $489.2 \mathrm{~Hz}$ for $\eta$, but the amplitude at $489.2 \mathrm{~Hz}$ is much larger than that at $978.4 \mathrm{~Hz}$ for $\Gamma$. Strong out-of-plane vibration with frequency of $489.2 \mathrm{~Hz}$ is generated, and as the result, the in-plane vibration is also excited at this frequency because of internal resonance. However, the frequency $980.5 \mathrm{~Hz}$ in the in-plane direction is not included in $\Gamma$. That is, the out-of-plane vibration draws in the in-plane vibration in a same period. Comparing Fig. 11(a) with Fig. 11(b), the amplitude of the out-of-plane vibration is much larger than that of the in-plane vibration. In Fig. 12, the waveforms of $\eta$ and $\Gamma$ at $274{ }^{\circ} \mathrm{C}$ are shown.

Figure 13 shows the maximum amplitudes $\eta$ and $\Gamma$ of periodic vibration, as obtained from the numerical computation in the disc temperature range of 20 to $350{ }^{\circ} \mathrm{C}$. Green and blue lines show the maximum amplitude of the periodic solutions obtained from the nonlinear analysis. Over the temperature range when only Type 1 is present in the linear analysis, both $\eta$ and $\Gamma$ have the same frequency of $980.5 \mathrm{~Hz}$ and the vibration amplitude of $\eta$ is approximately $0.5 \times 10^{4}$ times larger than that of $\Gamma$. This periodic solution corresponds to the squeal phenomenon that vibrates primarily in the in-plane direction along the disc surface. This squeal was actually observed in the experiments ${ }^{(1),(2)}$.

On the other hand, in the limited high temperature range in which Types 1 and 2 coexist in the linear analysis, the amplitude of $\Gamma$ on the blue line is much larger than that on the green line in the nonlinear analysis, whereas the amplitude of $\eta$ does not disappear but remains with amplitude slightly smaller than that on the green line. This phenomenon indicates the generation of chatter, which is coupled nonlinear vibration in the in-plane and out-of-plane directions due to internal resonance, because the frequency ratio of squeal in the in-plane unstable vibration to the out-of-plane unstable vibration of the system is fixed exactly at 2:1 (Fig. 11). The vibration characteristics are in good agreement with the experimental results ${ }^{(1),(2)}$ in the narrow high temperature range of 260 to $290{ }^{\circ} \mathrm{C}$. 


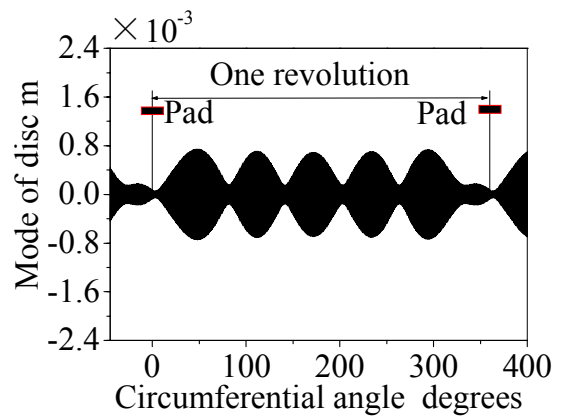

(a) Out-of-plane disc mode

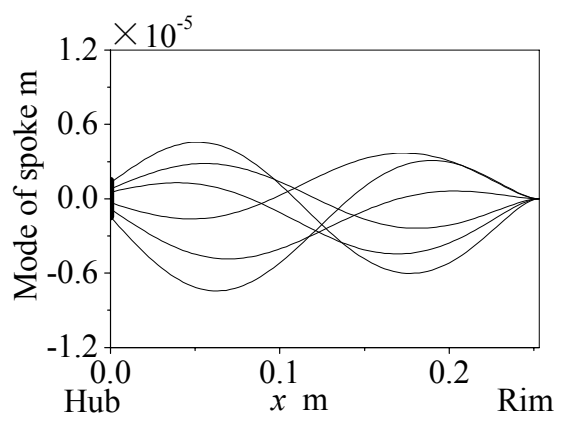

(b) Spokes on disc side

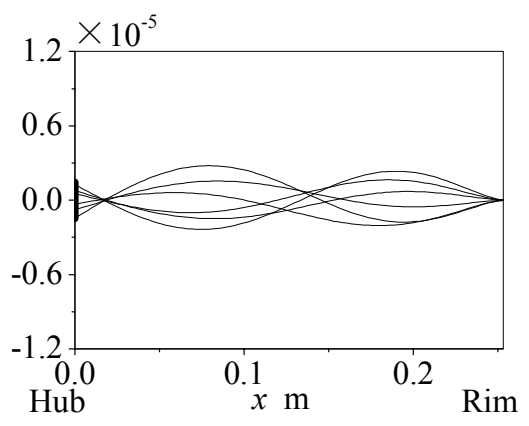

(c) Spokes on opposite side of disc

Fig. 14 Chatter modes of disc and spokes at $274{ }^{\circ} \mathrm{C}$

Consequently, in this narrow range of disc temperatures, Type 2 becomes unstable and is superimposed on Type 1 when Type 1 has already been generated in the linear analysis. As a coupled vibration, chatter in the out-of-plane and in-plane directions is generated through internal resonance. The resulting lower frequency coincides with the frequency of vibrationin the out-of-plane direction, and the higher frequency of vibration in the in-plane direction is altered such that it is only contained in the second-order harmonic component due to internal resonance. This behavior is the same as that of the vibrating system with three degrees of freedom analyzed in the previous section.

For the coefficient of friction $e_{0}=0.32$, the green branch (squeal) is partly unstable at around $274{ }^{\circ} \mathrm{C}$ within the disc temperature range in which the blue stable branch (chatter) exists. As disc temperature increases, the squeal of the green branch is generated first, and continues until the disc temperature reaches around $274{ }^{\circ} \mathrm{C}$. At that temperature, the jump phenomenon from squeal to chatter occurs suddenly. After that, at $288^{\circ} \mathrm{C}$ at the right end of the chatter range, the jump phenomenon from chatter to squeal occurs. Accordingly, the analytical results in Fig. 9 obtained from the linear analysis are not coincident with the results obtained from the nonlinear analysis (Fig. 13). The numerical results of nonlinear analysis are in good agreement with experiments ${ }^{(1),(2)}$.

As the coefficient of friction $e_{0}$ is increased from 0.32 , the unstable range of the squeal becomes wider and the chatter can be generated over a wider range of disc temperatures. In contrast, as the coefficient of friction $e_{0}$ is decreased from 0.32 , the unstable range of the squeal disappears gradually.

In Fig. 14, the chatter modes of the disc and the spokes on the disc side and the opposite side of disc at $274^{\circ} \mathrm{C}$ are shown. The chatter mode of the disc is coincident with the mode in the out-of-plane direction having five nodal radii (see Fig. 1), and the chatter mode of spokes on the disc side in the in-plane direction is almost in the second mode, which is shown at intervals of $1 / 6$ of the time period, and has large amplitude in comparison with the mode on the opposite side of disc. The displacement at $x=0.0 \mathrm{~m}$ on the horizontal axis corresponds to the circumferential displacement on the hub side caused by the torsional vibration of the disc and hub. In this manner, the computational results obtained from the 
nonlinear analysis differ from the results on Type 2 obtained from the linear analysis in Ref. (3), while the nonlinear analytical results are in good agreement with experiments ${ }^{(1),(2)}$. The bending moments in the spokes on the disc side may become very high according to the characteristic mode of the spokes under the boundary condition of fixed ends at the rim side as in Fig. 14. This is the reason why a wheel spoke can sometimes be broken if the chatter continues for a long time.

\section{Conclusions}

The chatter phenomena generated in the bicycle disc brake system were analyzed numerically taking the nonlinear frictional characteristics into account, and computational results were compared with experimental results. In order to further understand chatter, we also considered coupled nonlinear vibration due to the internal resonance between certain vibration modes generated in a simple system with three degrees of freedom subjected to friction. The results obtained are summarized as follows.

(1) In the linear analysis, two types of unstable vibrations occurred in the bicycle disc brake system. One was unstable in-plane vibration caused by dry friction independent of disc temperature. The other was unstable out-of-plane vibration generated in a limited range of disc temperatures caused by Coulomb friction. Within the narrow range in which both unstable vibrations coexisted, the frequency ratio between them was approximately, but not exactly, 2:1.

(2) The Runge-Kutta-Gill method was applied to the chatter phenomenon by using a bicycle disc brake model $^{(3)}$, taking into account the nonlinear frictional characteristics and the effects of increasing disc temperature during braking. Over a wide range of disc temperatures, squeal was mainly generated along the disc surface, similar to the results of the linear analysis. In addition, chatter was observed in a limited range of disc temperatures, in which the two unstable vibrations obtained from linear analysis coexisted, and the frequency ratio between them was completely fixed at 2:1. In this limited temperature range, the strong coupled in-plane and out-of-plane nonlinear vibration due to internal resonance occurred. As the result, the two unstable vibrations from the linear analysis generated in a limited range of disc temperatures could not occur individually because of the internal resonance, which is related to the nonlinear vibration phenomena.

(3) The numerical results obtained from the system with three degrees of freedom had many commonalities with the results obtained from the bicycle disc brake model.

(4) The numerical results obtained in this report were in a good agreement with experiments ${ }^{(1),(2)}$ with respect to the chatter frequency, the range of disc temperatures in which chatter occurred, and the chatter modes of the disc and hub, caliper and spokes.

\section{References}

(1) Ryu, T., Sueoka, A., Shirozu, K., Emura, A., Nakano, Y., Experimental Investigation on Squeal and Chatter Phenomena in Bicycle Disk Brakes (Part 1, Regeneration Experiments of Squeal and Chatter and Their Characteristics), Trans. of the Japan Society of Mechanical Engineers, Series C, (in Japanese), Vol.70, No.689, (2004), pp.30-37.

(2) Sueoka, A., Ryu, T., Shirozu, K., Emura, A., Nakano, Y., Experimental Investigation on Squeal and Chatter Phenomena in Bicycle Disk Brakes (Part 2, Generation Mechanisms of Squeal and Chatter and Their Countermeasures), Trans. of the Japan Society of Mechanical Engineers, Series C, (in Japanese), Vol.70, No.689, (2004), pp.38-45.

(3) Nakae, T., Sueoka, A., Ryu, T., Analytical Investigation on Squeal Phenomena Generated in Bicycle Disc Brakes, Journal of System Design and Dynamics, Vol.4, No.2, (2010), pp.379-390.

(4) Nakai, M., Yokoi, M., Fundamental Study on Squeal Noise of Wheel (Part 3, The Case of 
Squeal of a Circular Plate with Internal Resonance), Trans. of the Japan Society of Mechanical Engineers, Series C, (in Japanese), Vol.50, No.455, (1984), pp.1214-1224.

(5) Yasuda, K., Hayashi, N., Subharmonic Vibrations Generated in a Circular Plate Subjected to Initial Tension, Trans. of the Japan Society of Mechanical Engineers, Series C, (in Japanese), Vol.47, No.420, (1981), pp.987-999.

(6) Sakurai, A., Inoue, Y., Tsutsui, H., Itoh, H., Self-Excited Vibration Caused by CrossCoupling Force with Friction on Sliding Surface, Trans. of the Japan Society of Mechanical Engineers, Series C, (in Japanese), Vol.60, No.570, (1994), pp.380-385.

(7) JSME Mechanical Engineer's Handbook, Fundamentals, $\alpha 2$ : Dynamics of Machinery, (in Japanese), (2004), pp.53-69, The Japan Society of Mechanical Engineer.

(8) Theory of Vibration, Kyohei Kondo, (in Japanese), 5th revised edition, (2004), pp.195240, Baifukan. 